\title{
SOPHIA
}

PEER REVIEW JOURNAL

VISUAL SPACES OF CHANGE: UNVEILING THE PUBLICNESS OF URBAN SPACE

THROUGH PHOTOGRAPHY AND IMAGE

ISSN: 2183-8976 [PRINT] 2183-9468 [ONLINE]

Volume 4, Issue 1| Publication year: 2019

DOI 10.24840/2183-8976_2019-0004_0001_03

(c) SCOPIO EDITIONS

\section{HC (HORTUS CONCLUSUS)}

Written by Beate Gütschow

Translation: Jacqueline Todd

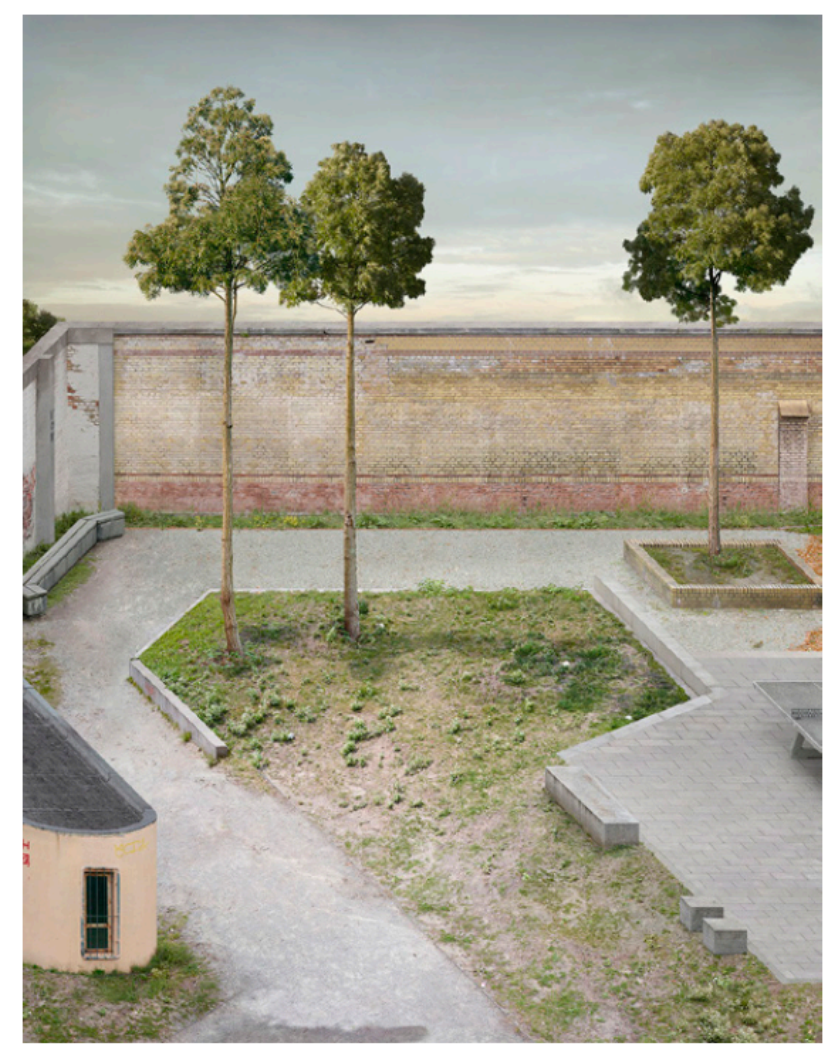

BEATE GÜTSCHOW

HC\#4, C-print, $148 \times 115 \mathrm{~cm}$ from the series "HC", 2018

HC stands for hortus conclusus, a Latin term that means "enclosed garden". In the $\mathrm{HC}$ series, the principles of medieval art with regard to pictorial space and perspective are applied to photography. In the Middle Ages, many images were rendered in parallel perspective; the viewpoint is always elevated. I have employed these spatial representations in my images. 


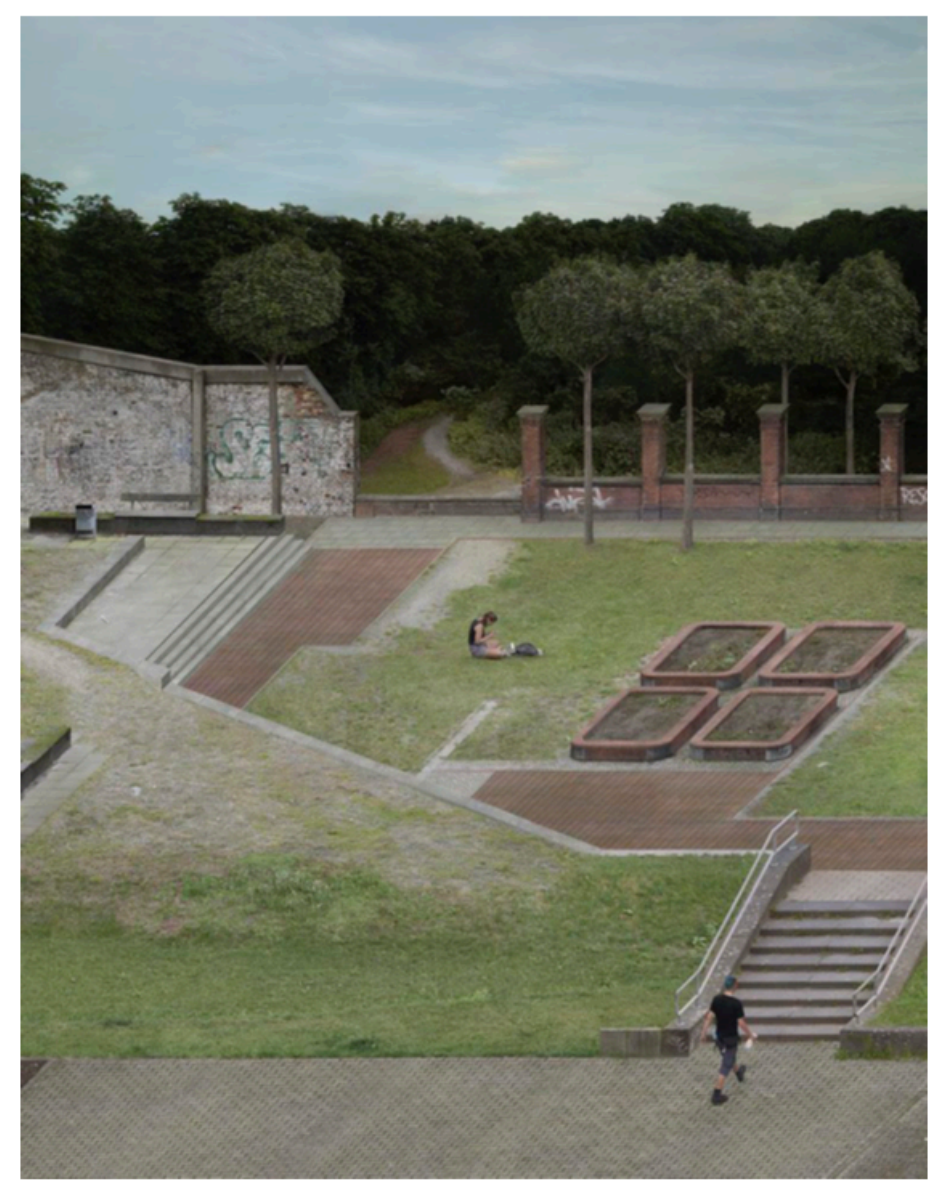

BEATE GÜTSCHOW

HC\#5, C-print, $148 \times 115 \mathrm{~cm}$ from the series "HC", 2018

You will find these principles in many images of gardens in medieval book illustrations. Linear perspective was not developed until the Late Middle Ages; prior to this, artists employed multiple perspectives. These did not obey a particular spatial logic; instead, the available perspectives served a narrative purpose. If the narrative required a scene to be set in a particular place, this space was "opened out", while the remaining space was shown as a flatter plane.

Valere Maxime, dits es faits memorables, 15. Century

In order to be able to represent architectural elements such as walls, fountains or benches in parallel perspective in my photographs, I had to take a roundabout route, which involved using a 3D software programme. I photographed the objects from all sides and used a software to assemble these photographs into one 3D object. This technic is called photogrammetry. Depending on the size of the object, 50 to 100 photographs may be required.

Using the photogrammetry programme Agisoft PhotoScan, a 3D model is generated from these photographs. The programme builds a mesh - a polygonal model that represents the object's dimensions. Then the photographic surface is mapped onto this mesh. In a 3D programme the perspective can be changed from linear perspective to parallel perspective. In 
my pictures all natural elements and human figures are taken from ordinary digital photographs. In Photoshop I combine the altered architectural elements with the figures and natural elements.

This picture combines two perspectival spaces. In parallel perspective, there should be no view into the distance, so no sky would be seen. As the viewpoint is elevated, we would be looking down onto grass, trees and architectural elements - all the way up to the top edge of the picture. In this image, as in most medieval book illustrations, a visual device is used: something is placed in the upper section of the picture to block the gaze, for example a wall or the edge of a forest; behind this, the sky opens up a new perspectival space where the viewing angle is much flatter.

There are various types of garden images in medieval book illustrations; these include religious images, images that accompany practical gardening instructions, and images featuring the motif of the "garden of love". In the religious images, every pictorial element has a particular significance. For example the hexagons appear in fountains, tables and borders. The hexagonal fountain symbolises the omnipotence of God'.

In parallel perspective, parallel lines in the image do not converge, they remain parallel. Although it looks as if the wall in this image widens towards the rear, this is an optical illusion, due to the fact that the eye is used to seeing converging lines in photographs. When working with photogrammetry I noticed following things:

The photogrammetric recording of an object can nevertheless be defined as photography. One aspect is new, however: when viewing these "photographs", there is no longer a single camera position. The viewer can choose in a 3D application a viewing angle retrospectively. They can even view the object from a vantage point that was not available when the photograph was taken, for example from a bird's-eye view.

1 Guillaume de Machaut, Le Remede de Fortune, 15. Century Anonymous, Garden of Paradise, around 1420-1420. 


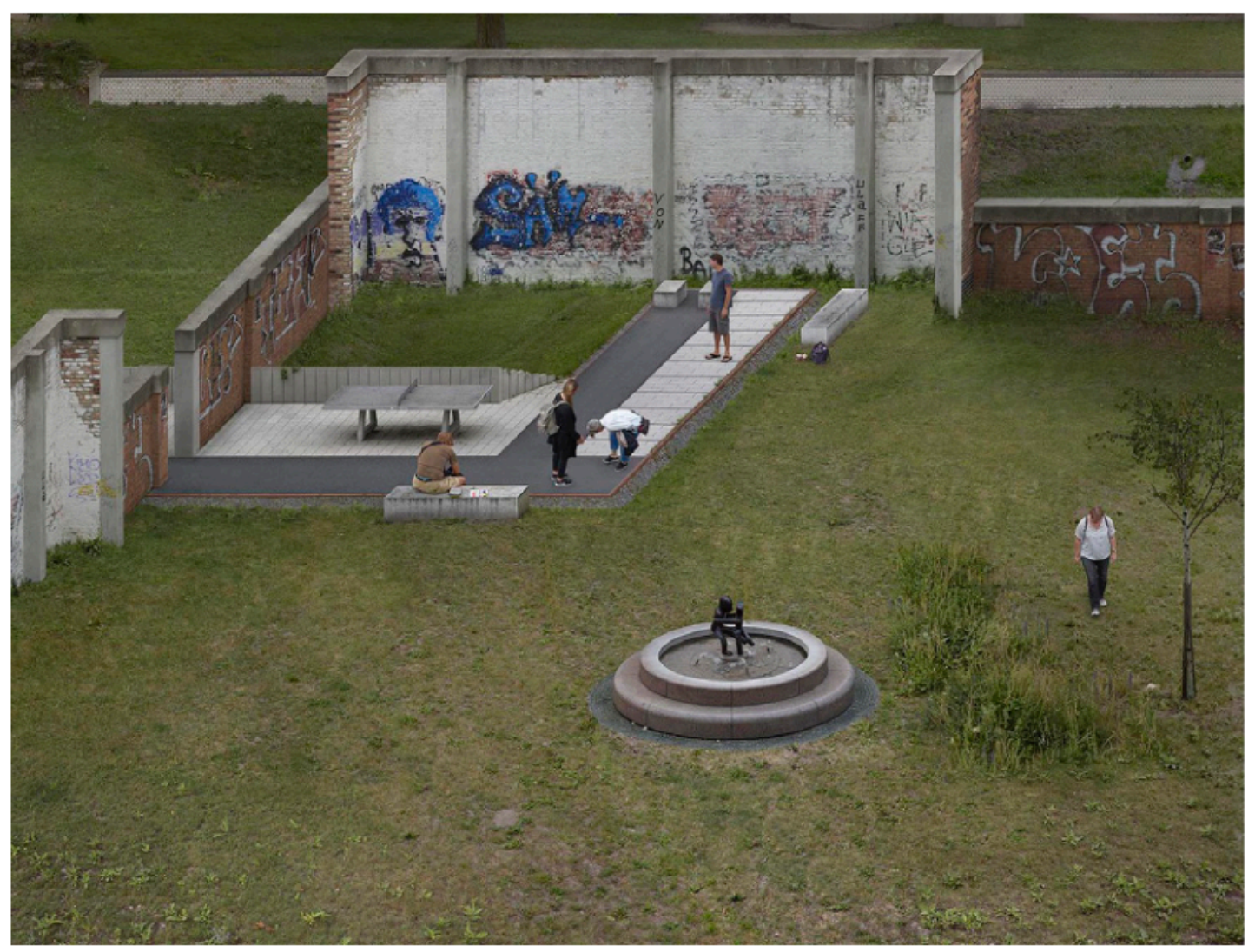

BEATE GÜTSCHOW

HC\#1, C-print, $115 \times 153 \mathrm{~cm}$ from the series "HC", 2018

I wonder whether this is a less dominant view of an object, precisely because the viewer can choose their own viewpoint? Does this circumstance generate greater knowledge? Or is it the other way around: does this method of capturing an image cause the viewer to have no relationship to the object, because the place where it was captured cannot be determined or understood?

Hans Belting talks about central perspective bringing the subject into the picture. The medieval image represents God, while the image in central perspective represents man. Wolfgang Ullrich, in turn, has described the transition from parallel perspective to central perspective as a depotentiation of the image.

In parallel and cavalier perspective, there is no fixed viewing position, nor does the object shrink when it is located further back in space.

Cavalier perspective is normally employed in planning and constructing, while central perspective has a long tradition of use in recording and capturing. In my work, the divergence between constructing and recording disappears - cavalier perspective is used here in the recording medium of photography.

Taking a photograph used to mean capturing something you knew had an interior; in the case of a house, this would be a load-bearing structure or a roof framework, for example. When you work with photogrammetry for a long time, however, you internalise the opposite 
aspect: the object is captured without its inner life. By working in three-dimensional space, you begin to see things more as outer shells, as filled objects. Photogrammetry therefore makes it possible to directly experience what photography has been doing for 150 years: namely, scanning a surface and remaining completely unaffected by the interior of the thing. An object is captured not from within, on the basis of its essence, but on the basis of its outer boundary. Photogrammetry makes this particularly evident through the shell-like representation of the object in space.
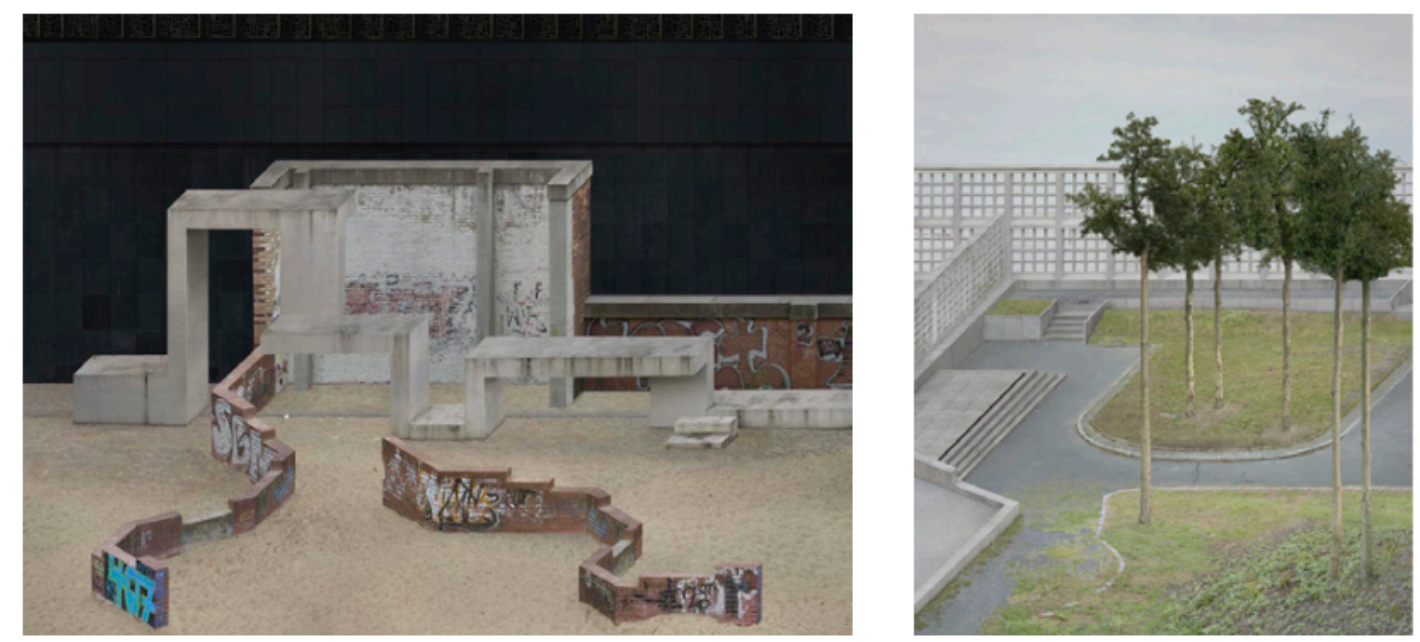

BEATE GÜTSCHOW

HC\#6, C-print, $115 \times 153 \mathrm{~cm}$ from the series "HC", 2018

HC\#8, C-print, $80 \times 64 \mathrm{~cm}$ from the series "HC", 2018

This article is a result of the project POCl-01-0145-FEDER-030605 - PTDC/ART-OUT/30605/2017 supported by Competitiveness and Internationalisation Operational Programme (POCI), under the PORTUGAL 2020 Partnership Agreement, through the European Regional Development Fund (ERDF) and through national funds by the FCT - Fundação para a Ciência e a Tecnologia. 